\title{
Alterstice
}

Revue internationale de la recherche interculturelle

International Journal of Intercultural Research

Revista International de la Investigacion Intercultural

\section{Dualité des processus de socialisation professionnelle des enseignants formés hors Québec : entre imposition et appropriation}

\author{
Joëlle Morrissette et Didier Demazière
}

Volume 8, numéro 1, 2018

URI : https://id.erudit.org/iderudit/1052611ar

DOI : https://doi.org/10.7202/1052611ar

Aller au sommaire du numéro

Éditeur(s)

Alterstice

ISSN

1923-919X (numérique)

Découvrir la revue

Citer cet article

Morrissette, J. \& Demazière, D. (2018). Dualité des processus de socialisation professionnelle des enseignants formés hors Québec : entre imposition et appropriation. Alterstice, 8(1), 95-106. https://doi.org/10.7202/1052611ar

\section{Résumé de l'article}

De plus en plus d'enseignants formés hors Québec intègrent les écoles québécoises. Ils y découvrent un contexte de travail très différent de celui où ils ont exercé précédemment. Cet écart est constitué ici comme observatoire d'une nouvelle socialisation aux manières de faire le métier, qui se réalise sur le terrain et par la pratique, pendant leurs premières expériences. Mobilisant une sociologie interactionniste des professions pour étudier cette socialisation en contexte de travail, nous avons conduit une recherche centrée sur leurs expériences d'enseignant dans leur pays d'accueil, en accordant une attention serrée à leurs interactions quotidiennes avec les membres de leur écologie professionnelle. Quatre enseignants formés hors Québec et certains de leurs partenaires de travail ont participé à des entretiens individuels et collectifs. L'analyse de ceux-ci a permis de dégager deux types de processus de socialisation. Chacun est associé à des acteurs privilégiés, mais non exclusifs : l'un procède par imposition de nouvelles manières de faire sous la pression d'acteurs dépositaires de capacités de sanction, l'autre procède par adhésion à de nouvelles manières de faire résultant de l'accompagnement d'acteurs qui transmettent des significations. L'explicitation de ces processus et l'analyse de leurs variations locales nous conduisent à discuter de la forte relation entre la socialisation professionnelle des enseignants formés hors Québec et les propriétés des contextes dans lesquels ils travaillent, incluant l'engagement de leurs partenaires. Finalement, cela met en exergue la fragilité de cette socialisation.
Ce document est protégé par la loi sur le droit d'auteur. L'utilisation des services d’Érudit (y compris la reproduction) est assujettie à sa politique d'utilisation que vous pouvez consulter en ligne.

https://apropos.erudit.org/fr/usagers/politique-dutilisation/ 


\title{
7
}

ARTICLE HORS THÈME

\section{Dualité des processus de socialisation professionnelle des enseignants formés hors Québec : entre imposition et appropriation}

\author{
Joëlle Morrissette ${ }^{1}$ et Didier Demazière ${ }^{2}$
}

\section{Résumé}

De plus en plus d'enseignants formés hors Québec intègrent les écoles québécoises. Ils y découvrent un contexte de travail très différent de celui où ils ont exercé précédemment. Cet écart est constitué ici comme observatoire d'une nouvelle socialisation aux manières de faire le métier, qui se réalise sur le terrain et par la pratique, pendant leurs premières expériences. Mobilisant une sociologie interactionniste des professions pour étudier cette socialisation en contexte de travail, nous avons conduit une recherche centrée sur leurs expériences d'enseignant dans leur pays d'accueil, en accordant une attention serrée à leurs interactions quotidiennes avec les membres de leur écologie professionnelle. Quatre enseignants formés hors Québec et certains de leurs partenaires de travail ont participé à des entretiens individuels et collectifs. L'analyse de ceux-ci a permis de dégager deux types de processus de socialisation. Chacun est associé à des acteurs privilégiés, mais non exclusifs : l'un procède par imposition de nouvelles manières de faire sous la pression d'acteurs dépositaires de capacités de sanction, l'autre procède par adhésion à de nouvelles manières de faire résultant de l'accompagnement d'acteurs qui transmettent des significations. L'explicitation de ces processus et l'analyse de leurs variations locales nous conduisent à discuter de la forte relation entre la socialisation professionnelle des enseignants formés hors Québec et les propriétés des contextes dans lesquels ils travaillent, incluant l'engagement de leurs partenaires. Finalement, cela met en exergue la fragilité de cette socialisation.

\section{Rattachement des auteurs \\ ${ }^{1}$ Université de Montréal, Montréal, Canada; ${ }^{2}$ Centre de sociologie des organisations, Science Po, Paris, France}

\section{Correspondance}

joelle.morrissette@umontreal.ca

\section{Mots clés}

enseignants formés hors Québec; socialisation; interactions; écologie professionnelle

\section{Pour citer cet article}

Morrissette, J. et Demazière, D. (2018). Dualité des processus de socialisation professionnelle des enseignants formés hors Québec : entre imposition et appropriation. Alterstice, 8(1), 95-106. 


\section{Introduction}

Comme le Canada, le Québec mène une politique active d'immigration afin de satisfaire ses besoins en maind'œuvre et de renouveler sa population décroissante (Zietsma, 2010). Ainsi, entre 1998 et 2009 , environ 3000 autorisations d'enseigner ont été délivrées à des immigrants, dont une majorité a débouché sur un recrutement dans les écoles québécoises (Niyubahwe, Makamurera et Jutras, 2014). Afin d'obtenir l'autorisation permanente d'enseigner, ces enseignants doivent valider 15 crédits de cours obligatoires dans l'université de leur choix. Ceci vise à les familiariser avec le système scolaire québécois, la didactique de l'enseignement des compétences disciplinaires et les modalités privilégiées pour les évaluer, ainsi qu'avec la gestion interactive d'un groupe-classe hétérogène sur le plan des performances et des origines ethnoculturelles. Cette formation est essentiellement théorique, et c'est donc en faisant de la suppléance dans les écoles, mais plus encore en décrochant un contrat pour faire leur période probatoire (2 ans) qu'ils (ré)apprennent à exercer leur métier dans les écoles québécoises.

Cette situation, associant expérience pratique acquise dans le pays d'origine et courte formation d'adaptation dans le pays d'accueil, présente des similarités avec celle des enseignants néophytes, débutant dans le métier. Car en dépit des différences dans leur parcours, ces deux catégories font la découverte du métier dans la mesure où toute activité professionnelle est éprouvée dans des contextes institutionnels, normatifs, culturels et sociaux (Demazière et Gadéa, 2009). Cependant, ce sont les enseignants débutants qui ont fait l'objet d'une attention particulière, afin d'analyser les modes d'intégration dans le métier et dans les établissements (Rayou et Van Zanten, 2004). Même si la socialisation professionnelle peut être considérée comme une composante constante du travail enseignant (Barrière, 2002), la période de découverte de la pratique du métier est un observatoire privilégié des processus de socialisation professionnelle. C'est pourquoi nous avons réalisé une enquête (CRSH 2015-2017) portant sur la socialisation dans les écoles québécoises des enseignants formés hors Québec.

\section{Problématique}

Les recherches qui explorent les expériences vécues par les enseignants soulignent le rôle des épreuves de début de carrière (Guibert et Périer, 2012). Dans cette perspective, la socialisation professionnelle est jalonnée par des événements inattendus et non anticipés, qui suscitent une déstabilisation des jeunes enseignants (Van Zanten, 1999). C'est que ceux-ci ont souvent une vision idéalisée de leur métier, constituée à travers une " socialisation spécifique " et " anticipatrice ", ancrée dans les expériences de parents eux-mêmes enseignants (Charles et Clément, 1997), inspirée des figures d'enseignants connus en tant qu'élève (Perrenoud, 1996) ou nourrie d'engagements dans des activités d'animation de jeunes (Charles et Legendre, 2006). Et cette socialisation présente des écarts, plus ou moins larges, avec les situations concrètes rencontrées. Nous reprenons et transposons pour les enseignants formés hors Québec cette idée d'écarts entre le modèle professionnel, acquis, et la réalité, nouvelle, ainsi que la nécessité qui en découle d’opérer des « deuils professionnels » (Barrère, 2002).

Les expériences d'intégration des enseignants formés hors Québec dans les écoles de leur société d'accueil a fait l'objet d'un certain nombre de recherches. Deux recensions récentes soulignent des résultats convergents, concernant surtout les difficultés rencontrées sur le plan de l'accès au marché du travail et des démarches administratives, des pratiques pédagogiques et des relations au travail (Morrissette, Charara, Boily et Diédhiou, 2016; Niyubahwe, Mukamurera et Jutras, 2013). Certains auteurs ont dégagé des modes différenciés d'acculturation (assimilation, séparation, etc.) d'immigrants - pas exclusivement enseignants - dans de nouveaux contextes socioculturels (Berry et Sabatier, 2011; Camilleri, 1989; Kanouté, 1999). D’autres ont décomposé les étapes traversées par les immigrants, par exemple des enseignants ayant intégré des écoles de la région de Sherbrooke (Niyubahwe, Mukamurera et Jutras, 2013) : choc de la réalité, perte des repères culturels, désenchantement, développement de stratégies de survie, adaptation progressive (voir également Duchesne, 2010). Dans cette veine, les adaptations repérées chez les nouveaux arrivants sont saisies comme des transformations identitaires (Deters, 2006; Laghzaoui, 2011). Des travaux centrés sur les stratégies des enseignants immigrants ont souligné l'importance de l'engagement dans des activités de familiarisation à leur société d'accueil (Charles et Legendre, 2006; Duchesne, 2008; Jabouin et Duchesne, 2012; Phillion, 2003) ou montré une variété de conduites : la négation, la banalisation, le retour sur soi (la remise en question) et l'affrontement (Lefebvre, 2011). 
Dans l'ensemble de ces travaux, l'accent est porté sur l'individu, sa personnalité et ses attitudes, l'analyse se centrant sur les processus psychologiques transformatifs qui traversent les expériences d'acculturation interculturelle. Sauf exception (voir Morrissette et collab., 2016), ils accordent une place limitée aux environnements de travail et aux interactions qui s'y déploient. Or nous faisons l'hypothèse que ces interactions influencent directement l'intégration. Et en les prenant en compte, nous voulons placer la socialisation professionnelle des enseignants formés hors Québec au cœur des situations de travail.

\section{La socialisation professionnelle en contexte}

Pour étudier les dynamiques du rapport au métier des enseignants formés hors Québec au cœur des situations de travail, nous retenons une approche issue de la sociologie interactionniste des professions (Baszanger, 1986; Bucher et Strauss, 1961; Freidson, 1970) selon laquelle la socialisation professionnelle s'étend au-delà de l'assimilation des savoirs spécialisés nécessaires pour maîtriser une expertise et des périodes de formation préalables à l'exercice professionnel. Celui-ci exige de s'approprier des visions partagées du métier et d'assimiler une conception spécifique des problèmes à résoudre et des manières d'y remédier (Becker, Geer, Hughes et Strauss, 1961; Hughes, 1955), ce qui passe par l'expérimentation concrète des conditions de réalisation de l'activité et par des échanges avec les partenaires de travail (Becker, 1963). Ainsi, ce n'est pas tant la sanction officielle et externe qui compte que la reconnaissance et la sanction interne, et ce, dans un double sens: celle du groupe de pairs qui accepte comme membre un nouvel entrant, et celle des autres acteurs (et autres groupes) avec lesquels ils interagissent dans des situations de travail (Guignon, 2012).

Cette socialisation professionnelle qui forge un rapport au métier est ainsi le produit d'interactions situées. Comme le soutient Baszanger (1981) à propos des médecins, dans les institutions de formation, les contextes de travail sont peu pris en compte : ils sont considérés comme des espaces secondaires, homogènes, et la vision du métier qui est enseignée est " tenue pour partagée ", taken-as-shared (Cobb et Bauersfeld, 1995). Par analogie, les enseignants doivent "faire leurs classes " (Guibert, Lazuech et Rimbert, 2008). Toute pratique professionnelle s'actualise dans une variété de contextes qui doivent être pris en compte pour saisir la dynamique de socialisation. Cette diversité peut être saisie de multiples manières : conditions statutaires (suppléance, contrat, poste), institutionnelles (école privée/publique), organisationnelles (fonctionnement de l'école et position dans la segmentation scolaire), mais aussi relationnelles (configuration des partenaires de travail). C'est cette dimension que nous privilégions, en considérant la variété des partenaires de travail et leur « définition de la situation » (Thomas, 1923), c'est-à-dire la façon dont ils interprètent les situations dans lesquelles ils se trouvent et donc les cadrent.

Et comme l'indiquent Becker et McCall (1990), la définition de chacun est articulée à celles des partenaires de travail. S'engagent alors des ajustements mutuels, qui contribuent à donner du sens aux situations et à cadrer les pratiques. L'analyse de ces processus exige de prendre en compte les positions respectives de ces acteurs, car celles-ci sont inscrites dans des asymétries de statut, de pouvoir et de légitimité. Ainsi les enseignants formés hors Québec, qui débutent dans les écoles québécoises même s'ils sont expérimentés, se trouvent en situation de découverte d'un nouveau milieu de travail, de ses valeurs, ses codes, ses conventions, ce qui les exposent à des faux-pas. Et ils interagissent avec des partenaires qui ont acquis une familiarité de ce milieu. C'est dans cette asymétrie que des relations se nouent et que la socialisation professionnelle se développe.

\section{Méthodologie}

Considérant ces appuis théoriques, nous avons réalisé une enquête auprès de quatre enseignants formés hors Québec travaillant en école secondaire et ayant intégré des écoles montréalaises depuis quelques années ainsi qu'auprès d'autres acteurs de leur environnement de travail : deux conseillers pédagogiques, une directrice d'école et un parent d'élèves. Nous les avons invités à signer un formulaire de consentement éthique garantissant la confidentialité et l'anonymat de leurs propos, en relation avec la tenue de cinq entretiens de groupe (EG) dans le cadre desquels les enseignants ont pu raconter leurs expériences dans l'école québécoise comme dans leur pays d'origine et expliciter des épisodes de turbulence avec leur nouvel entourage. Leurs partenaires ont été associés aux trois derniers entretiens, afin qu'ils commentent ces épisodes et échangent avec les enseignants. Cela permet de révéler des négociations et cadrages au sujet de ces frictions, de favoriser l'explicitation des cadres normatifs tacites du travail enseignant et de définir la socialisation en termes d'apprentissages et d'ajustements. La ficelle 
méthodologique de la comparaison (Becker, 2013) a été employée pour favoriser l'émergence de catégories d'interprétation, dont les participants se sont saisis in situ pour approfondir la co-analyse des expériences différenciées. Ces matériaux ont été complétés par des entretiens individuels (EI) à orientation biographique (Demazière, 2011) conduits auprès des enseignants, afin d'inscrire le récit de leur pratique professionnelle au Québec dans leur parcours et leurs expériences de formation, de travail et de migration.

Nous avons privilégié un grain fin d'analyse, en étant attentifs aux relations que ces enseignants ont nouées avec leurs partenaires de travail et avec les élèves et en focalisant l'analyse sur les expériences relatives à leur première année dans les écoles montréalaises. Dans cette optique, les transcriptions des entretiens ont été exploitées selon une approche analytique centrée sur les interactions, l'objectif étant de comprendre comment les enseignants formés hors Québec ajustent leurs pratiques et transforment leur conception du métier à travers les interactions dans lesquelles ils sont engagés. Dans l'exploitation des matériaux, nous avons examiné les événements vécus comme des surprises par les enseignants, identifiés comme des difficultés rencontrées et pouvant être considérés par leurs partenaires comme des "erreurs dans le travail " (Hughes, 1951). Nous avons pris ces événements comme des révélateurs de processus plus diffus d'une socialisation professionnelle en actes, en contexte. Ce tableau fournit quelques informations sur les enquêtés, nécessaires à la bonne compréhension des analyses (afin de garantir l'anonymat, et outre le recours à des prénoms fictifs, quelques informations ont été à dessein modifiées).

Tableau 1. Profil des enseignants

\begin{tabular}{|l|l|l|l|l|}
\hline $\begin{array}{l}\text { Noms } \\
\text { (fictifs) }\end{array}$ & $\begin{array}{l}\text { Pays } \\
\text { d'origine }\end{array}$ & $\begin{array}{l}\text { Formation } \\
\text { initiale }\end{array}$ & $\begin{array}{l}\text { Expérience } \\
\text { dans le pays } \\
\text { d'origine }\end{array}$ & $\begin{array}{l}\text { Contexte de la première année d'expérience } \\
\text { d'enseignement au Québec }\end{array}$ \\
\hline Anna & Moldavie & $\begin{array}{l}\text { Licence en } \\
\text { anglais } \\
\text { langue } \\
\text { étrangère }\end{array}$ & 3 ans & $\begin{array}{l}\text { Arrivant au Québec en 2007, elle suit un programme de } \\
\text { formation en enseignement à l'Université Concordia et } \\
\text { obtient son diplôme en 2010. Elle décroche trois petits } \\
\text { contrats en enseignement de l'anglais langue seconde } \\
\text { dans trois écoles différentes, totalisant 64 \% de tâches } \\
\text { pour cette 1 année. }\end{array}$ \\
\hline Rebiha & Algérie & $\begin{array}{l}\text { Licence } \\
\text { d'enseigne- } \\
\text { ment en } \\
\text { mathémati- } \\
\text { ques }\end{array}$ & 13 ans & $\begin{array}{l}\text { Arrivant à Montréal en 2010, elle obtient un contrat } \\
\text { d'enseignement dans son domaine dans une école } \\
\text { privée secondaire avec élèves sélectionnés. Elle réalise } \\
\text { en concomitance les 15 crédits de cours universitaires } \\
\text { obligatoires et trois stages optionnels. }\end{array}$ \\
\hline Naïma & Algérie & $\begin{array}{l}\text { Licence en } \\
\text { français } \\
\text { langue } \\
\text { seconde }\end{array}$ & $\begin{array}{l}\text { Immigrant au Québec en 2012, elle commence } \\
\text { rapidement les 15 crédits de cours et décroche très vite } \\
\text { un contrat de 9 mois dans son domaine, dans une école } \\
\text { publique secondaire située en milieu défavorisé. Au } \\
\text { cours de l'année, elle est la 8 enseignante responsable } \\
\text { de ces groupes d'élèves. }\end{array}$ \\
\hline Faïza & Tunisie & $\begin{array}{l}\text { Maîtrise en } \\
\text { sciences } \\
\text { naturelles }\end{array}$ & $\begin{array}{l}\text { Arrivant au Québec en 2010, elle fait 2 ans de bénévolat } \\
\text { auprès d'enfants ayant des difficultés d'apprentissage. } \\
\text { Puis elle commence la suppléance dans les écoles en } \\
\text { même temps qu'elle suit les 15 crédits de cours et } \\
\text { débute des stages optionnels. Elle décroche un contrat } \\
\text { de 25\% en cours d'année pour enseigner à un groupe } \\
\text { de science et technologie de secondaire 4, et } \\
\text { abandonne ses stages. }\end{array}$ \\
\hline
\end{tabular}




\section{Les processus de socialisation professionnelle}

Les récits que les enseignants formés hors Québec font de leur première année d'expérience dans les écoles québécoises sont particulièrement riches en événements et anecdotes. De cet amoncellement de petits faits, nous avons produit une description détaillée des occurrences contribuant à leur socialisation professionnelle, c'est-à-dire à des changements dans leurs manières d'enseigner et de concevoir leur métier. Nous en avons dégagé deux processus typiques : un processus d'adaptation des pratiques de travail sous la contrainte de situations et d'acteurs qui exercent pressions ou sanctions et un processus d'appropriation de nouvelles significations délivrées par des acteurs qui les soutiennent en explicitant les attentes. À partir d'une sélection de " chocs " vécus par les enseignants et pertinents pour expliciter les composantes élémentaires de ces processus transversaux, nous montrons comment ceux-ci sont modulés en fonction des contextes de travail.

\section{La socialisation par imposition et obligation : des variations contextuelles limitées}

Important leurs façons de travailler dans les écoles québécoises, les enseignants formés hors Québec se heurtent rapidement à un ensemble de résistances, d'abord des élèves. Confrontés à des situations de blocage qui les empêchent de travailler, ils sont contraints d'adapter leurs pratiques. Ce processus est d'autant plus implacable que les plaintes des élèves sont souvent relayées par les parents qui exercent alors une pression directe sur les enseignants, et que les directions d'école font valoir, au moins indirectement, un pouvoir de sanction d'autant plus menaçant que les enseignants ont des statuts précaires.

Les relations avec les élèves et la gestion de la classe sont des points focaux des récits d'apprentissage, un apprentissage à marche forcée, émaillé de faux-pas que les enseignants n'ont d'autre choix que de corriger rapidement. Les quatre enseignants interrogés ont fait la même expérience, indépendamment de leur contexte de travail. Dès les premiers jours de classe, ils reproduisent les rapports de place dont ils ont l'habitude : verticaux, définis par l'autorité de l'enseignant et la crainte des élèves, fixant une distance marquée entre eux. Par leurs attitudes de froideur, de fermeté et d'inflexibilité, ils cherchent à maintenir les élèves à distance, à instaurer le calme dans la classe et à fonder les bases du travail enseignant tel qu'ils l'ont pratiqué auparavant. Même quand ils ont suivi des stages sur les pédagogies actives au Québec, ils ont reproduit l'enseignement magistral et familier. Habitués à professer pour les " élites » devant un groupe homogène et indifférencié, ils tentent d'ignorer les élèves ayant des difficultés d'apprentissage ou ceux qui ont des problématiques particulières (autisme, déficit d'attention, etc.), vus comme des " handicapés » (Rebiha).

Ces manières de faire sont si éloignées des attentes des élèves qu'elles génèrent des blocages : les élèves ne répondent pas aux consignes, chahutent, contestent, refusent de travailler. Cette indiscipline peut se traduire parfois, en fonction des caractéristiques des classes, par des conduites exacerbées : " lls piquaient des crises ", et même l'un d'entre eux " a ouvert la fenêtre, il m'a insultée, et est sorti dans la rue " (Naïma). Les enseignants subissent durement l'épreuve du terrain: "Ils me testent sur tous les points: sur la patience, sur l'entente, est-ce que je comprends ou non. J'ai été insultée, mais alors de très belles insultes! » (Faïza). Devant ces turbulences et le refus d'obtempérer des élèves, c'est leur professionnalité qui est mise en échec : "I'ai perdu mes moyens » (Faïza). II y a alors urgence à s'adapter.

Mis à part des manifestations d'indiscipline, les élèves procèdent à des cadrages puissants du travail enseignant, infléchissant les pratiques dans une série de dimensions importantes. Dans les quatre expériences étudiées ici, les élèves cherchent à casser les codes d'une relation formelle et verticale, en multipliant les questions d'ordre personnel, sur la vie familiale ou les loisirs de leur enseignant, en engageant des interactions familières (demander à faire des selfies par exemple). Perçues comme des intrusions dans leur vie privée, ces attitudes sont réinterprétées comme des moyens de gérer le climat de la classe et d'instaurer des conditions propices à l'enseignement : " J'ai trouvé une façon de faire, c'était d'être leur amie » (Faïza). Ici les ajustements se font parfois de manière très rugueuse, comme l'indique Anna, racontant un épisode où elle regardait un élève qui n'était pas attentif : " Je l'ai juste regardé dans les yeux; "pourquoi vous me regardez comme ça?" et j'ai dit "parce que j'ai mes yeux et j'ai le droit de te regarder"; il me dit "vous m'intimidez si vous me regardez comme ça"; [...] le mot intimidation semble être le premier mot qu'un élève d'ici commence à dire (Anna) ». 
Un autre point de tension porte sur le travail des élèves et les exigences de l'enseignant. Ici la pression est souvent directe et forte quand les élèves estiment que les charges de travail sont trop lourdes. Les enseignants découvrent que les élèves ont de fortes capacités d'inertie et de résistance : " Les élèves, déjà, pour prendre un stylo et écrire deux phrases [...] pour donner un devoir d'une page: "Mais madame, pourquoi on écrit 300 mots ? On écrit juste un paragraphe, c'est suffisant" » (Naïma). N'acceptant pas cette situation, Naïma tente de résister, mais s'engage alors un rapport de forces où elle est renvoyée à une sorte de médiocrité professionnelle : "Les élèves me disent: "Ah madame Christine, elle, une bonne enseignante !" Je demande: "Mais pourquoi madame Christine est-elle bonne ?" Ils répondent: "Parce qu'avec elle, le vendredi, c'est relax; on discute, on fait ce qu'on veut" ". À contrecœur, elle finit par céder, notamment pour préserver sa réputation auprès d'une direction d'école attentive aux avis des élèves.

Le contrôle des élèves sur les charges de travail apparaît plus faible dans le cas de Rebiha, qui enseigne dans une école privée secondaire où les élèves sont sélectionnés. Pour elle, ce sont les parents qui formulent des exigences spécifiques pour leur propre enfant : "II fallait travailler suivant les exigences de chaque parent : avec cet élève, il fallait que je me comporte comme ça parce que son père attend de moi telle chose ». Les pressions des parents lui apparaissent comme une intrusion illégitime dans son exercice professionnel, ce que soulignent les autres enseignants formés hors Québec rompus à des systèmes éducatifs où les parents "n'ont rien à faire ou à dire dans le milieu scolaire " (Rebiha). Mais elle n'a d'autre choix que de céder et de s'ajuster en individualisant ses interventions. Dès lors le jeu se calme en classe, les parents, satisfaits, se révèlent des alliés qui incitent leurs enfants à s'investir davantage dans leurs travaux scolaires. Ici la valorisation partagée par l'enseignant et les parents des performances scolaires facilitent les adaptations. Dans d'autres contextes, les relations avec les parents sont moins pacifiées, notamment quand ils répercutent les plaintes de leur enfant auprès de l'enseignant, mais aussi auprès de la hiérarchie s'ils estiment que leur intervention directe a été inefficace.

Les enseignants formés hors Québec sont d'autant plus sensibles à cette éventualité - qui est parfois une menace explicite - qu'ils sont habitués à devoir présenter une image irréprochable à leur hiérarchie. Moins qu'une ressource, celle-ci est perçue comme une menace latente qu'il vaut mieux tenir à distance : "Ma directriceadjointe, je la craignais un tout petit peu [...] Donc je n'osais pas. Si elle me croisait pour me dire "Alors, comment ça va ?", automatiquement je disais "Tout va bien" » (Faïza). Dès lors les passages de la direction dans la classe apparaissent comme un contrôle et une possibilité d'évaluation négative, ce qui provoque des alignements sur les commentaires énoncés. Ainsi Naïma s'efforce de développer une relation " plus chaleureuse " avec les élèves après que la directrice-adjointe lui " a dit "Iorsque tu es avec tes élèves, tu es comme ça : écrivez ! Faites ceci, faites cela! [...] Tu souris juste le strict minimum" ". La puissance du contrôle hiérarchique est d'intensité variable selon les écoles, et elle est parfois renforcée, dans un contexte de fragilité d'emploi, par les conduites de collègues, comme le rapporte Faïza : "Lors de mon premier contrat [...] mon collègue est venu une fois me dire "Écoute la direction est venue me parler de toi, me demander après toi" [...] J'ai compris que la direction essayait de comprendre comment ça fonctionne avec la nouvelle à travers les anciens collègues ».

La force des processus de socialisation par imposition et obligation se déploie dans l'écart initial entre les expériences des enseignants formés hors Québec et leurs nouveaux contextes de travail. Elle résulte de l'ampleur des réactions d'acteurs qui ont une capacité de sanction, immédiate dans le cas des élèves. Sous la pression de ces derniers comme des parents et directions, des adaptations rapides sont faites. Si les exigences imposées aux enseignants varient selon les contextes, en particulier avec les caractéristiques d'hétérogénéité des classes (multiculturalisme, problématiques dysfonctionnelles, résultats scolaires), la nécessité d'aligner ses pratiques est une contrainte générale. Le second processus de socialisation professionnelle est beaucoup plus dépendant des contextes locaux.

\section{La socialisation par compréhension et adhésion : de fortes variations contextuelles}

Dans ces contextes, les enseignants formés hors Québec ne sont pas seulement confrontés à des acteurs engagés dans l'exercice d'un pouvoir de configuration de leurs pratiques professionnelles. Ils sont aussi inscrits dans des relations moins verticales ou déséquilibrées et peuvent, potentiellement, bénéficier de soutiens et d'accompagnements délivrés par leurs partenaires de travail. Dans ces interactions se développe un autre type de 
socialisation professionnelle, fondée sur l'explicitation des significations associées aux situations rencontrées et sur la production d'une interprétation partagée. L'enquête révèle que les contextes, caractérisables par la densité des partenaires de travail et leur engagement dans cette intercompréhension, sont fortement contrastés. À un pôle, ces partenaires sont nombreux et actifs, formant un contexte porteur pour la socialisation, à l'opposé ils sont quasiment absents et les enseignants sont alors renvoyés à leurs ressources personnelles, et à l'échelle intermédiaire les appuis relationnels sont épars et correspondent à des rôles institutionnalisés.

Incontestablement, c'est Rebiha qui a bénéficié de l'entourage professionnel le plus fourni et actif pour lui donner les clés de compréhension du travail enseignant au Québec. Elle mentionne en effet pêle-mêle la secrétaire de l'école, le concierge, une enseignante immigrée, un orthopédagogue, un technicien en éducation spécialisée, la directrice de l'école, un conseiller pédagogique, qui sont intervenus pour l'aider à se repérer et à s'intégrer dans son nouvel univers professionnel. Rebiha est incitée à solliciter la secrétaire et le concierge pour se familiariser avec le fonctionnement de l'école (ex : gestion des absences) et à s'informer d'aspects de son métier qui n'existent pas dans son pays d'origine, tels que le suivi administratif individualisé des élèves en difficulté ou la surveillance des élèves hors classe. Elle noue une relation de confiance avec la secrétaire, qui lui permet de la solliciter pour gérer des situations - même pédagogiques - qu'elle perçoit comme illégitimes ou ahurissantes. Par exemple, grâce à ses explications, son étonnement face à l'exigence des élèves de disposer de leurs notes de cours pour un examen cède la place à la compréhension de cette règle au regard de l'approche par compétences qui privilégie la mobilisation originale de ressources par l'élève sur la mémorisation des savoirs.

Elle est aussi épaulée par un conseiller pédagogique qui l'initie à l'évaluation critériée, ce qui l'amène à valoriser la démarche suivie par l'élève même si la réponse finale est erronée. Elle modifie alors ses pratiques de notation : "J'ai repris mes copies et ai recommencé la correction. Là j'ai appris ». Elle reçoit aussi l'appui de spécialistes d'éducation spécialisée, qui l'informent de problématiques qu'elle découvre (autisme, déficit d'attention, dyscalculie, etc.), lui conseillent des lectures, échangent avec elle sur ses expériences auprès de ce type d'élèves. Cela lui donne de l'assurance, non seulement en classe mais aussi à l'égard des parents avec qui elle se sent capable de dialoguer de façon plus sensible et contrôlée. La direction œuvre dans le même sens, en explicitant la logique inclusive centrale dans l'école québécoise, si opposée au système méritocratique jadis connu. Elle découvre ainsi l'intérêt de prendre en compte les besoins particuliers de chaque catégorie d'élèves, et le plaisir de les voir progresser par rapport à des objectifs adaptés à leur situation.

Enfin d'autres appuis sont plus informels, comme quand certains élèves lui apprennent quelles mesures particulières étaient adoptées par ses prédécesseurs pour les élèves intégrés : par exemple, ils lui expliquent I'horaire adapté qui convient pour leur camarade autiste. Rebiha se lie aussi avec Miranda, une enseignante ayant immigré antérieurement. Dès les premières semaines, celle-ci décode avec elle les événements de classe qui l'ont choquée ou surprise, l'initie aux règles tacites de la vie sociale entre collègues (ex : participer à l'organisation de petites fêtes) et l'instruit des actualités et enjeux de la société québécoise afin de faciliter sa participation active aux discussions de l'équipe-école. Elle la guide aussi dans ses démarches auprès du ministère pour obtenir son permis d'enseigner, lui révélant des informations que la direction d'école lui cachait afin de bloquer sa mobilité dans un contexte de pénurie d'enseignants en mathématiques. Inscrite dans un contexte favorisant une socialisation par compréhension et adhésion, Rebiha confesse que pour la première fois de sa carrière, elle est détendue et sereine au travail, et qu'elle éprouve du plaisir à enseigner : " Je ne me vois pas partir ailleurs ".

Faïza connaît un contexte inverse, où elle ne bénéficie guère du soutien de ses partenaires de travail. Du fait de son relatif isolement, elle peine à comprendre et gérer certains événements de la classe, comme les moqueries des élèves, suscitées par le fait qu'elle employait " des mots qui n'étaient pas compris " et renforcées par sa manière autoritaire de réclamer le silence. C'est alors sa propre fille, elle-même socialisée par sa scolarité à Montréal, qui l'aide à reconstruire le sens de sa journée: " J'avais un bon professeur à la maison, c'était ma fille [...] lorsque je rentrais à la maison, je lui demandais des explications sur certaines choses et elle m'expliquait ". Parvenant graduellement à décoder les situations, Faïza s'ajuste et privilégie des interventions plus contenues et polies auprès des élèves. Dans cette dynamique, certains lui délivrent quelques conseils sur les manières usuelles de gérer ceux qui perturbent, et de son côté elle essaie de " cibler certains élèves qui pouvaient m'aider par rapport à la gestion de la classe ". C'est avant tout sur des ressources personnelles plutôt qu'institutionnelles que Faïza s'appuie. II en 
va de même quand elle évoque un collègue qu'elle consulte et qui l'aide à comprendre les attentes locales pour éviter d'autres sanctions. Par exemple, face aux absences répétées d'un élève, il lui suggère de prévenir les parents pour mieux comprendre la nature du problème, ce qu'elle n'a jamais fait dans son pays d'origine. Elle découvre ainsi les attentes de collaboration avec eux, centrale dans son nouveau contexte de travail. Concernant les évaluations des élèves, ce collègue juge qu'elle note trop sévèrement, que ses examens son trop difficiles, qu'il y a trop d'élèves en échec, qu'elle doit attacher plus d'importance à leur progression, autant de conseils qui la conduisent à modifier ses pratiques, pour obtenir des moyennes similaires à celles de ce pair et s'éviter des problèmes potentiels avec la direction. Faïza ajuste donc ses pratiques au coup par coup, en fonction de conseils dispersés, et rares, qu'elle reçoit, et elle peine à s'approprier les manières d'enseigner au Québec, faute d'une solide transmission des significations sous-jacentes.

Le type intermédiaire de contexte local, plutôt caractéristique des expériences de Naïma et d'Anna, se caractérise par des soutiens institutionnalisés fournis par des partenaires de travail, mais en faible nombre : dans les deux cas, il s'agit de conseillers pédagogiques et de directions d'école. Les premiers ont proposé des aides concrètes, dans la construction des leçons et la programmation de la progression des apprentissages comme dans leur évaluation. Dans ces domaines, il s'agit d'infléchir des pratiques opératoires dans les pays d'origine, mais qui suscitent des difficultés dans la classe parce que les évaluations des élèves s'apparentent à des sanctions trop sévères et que la pédagogie mobilisée vise surtout l'assimilation de savoirs. Les conseillers explicitent la logique d'ensemble des apprentissages, avec ses cycles pluriannuels et les attentes de fin de cycle, avec ses principes d'évaluation critériée également. Mais leur action reste isolée et elle n'enclenche qu'une adhésion superficielle de Naïma comme d'Anna, comme l'illustre le cas des notes : d'un côté elles n'estiment plus que les notes sont " gonflées " artificiellement, et d'un autre côté elles persistent à penser que les erreurs devraient demeurer au centre de l'évaluation.

Isolées de leurs collègues pour des raisons différentes (Naïma a eu des problèmes initiaux d'intercompréhension et déjeune seule dans sa classe, et Anna qui a une charge fragmentée est la seule spécialiste de l'anglais dans ses différents lieux de travail), elles n'ont pas l'opportunité d'échanger avec des pairs pour recadrer leur définition de la situation. Néanmoins, elles infléchissent leurs pratiques en prenant conscience que la sévérité des notes délivrées n'est pas un signe de qualité ou de prestige professionnels, mais est, à rebours, vue comme une marque de disqualification et de dévalorisation professionnelles : "C'est pour ça que les élèves disent que je suis mauvaise. C'est pour ça qu'ils disent que je ne suis pas bonne, que je suis méchante " (Naïma). Les directions d'école ont un rôle assez limité, qui consiste à souligner les avantages concrets de certaines pratiques, notamment dans la relation pédagogique et la gestion de classe. Elles valorisent aussi les adaptations observées, dispensant des validations ("Elle est venue me voir pour dire que c'était bien », Naïma), des félicitations en qualifiant de " prouesse " le fait de réussir à capter l'attention soutenue des élèves (Naïma), ou des encouragements (Anna). Ces marques de reconnaissance soutiennent les transformations qui s'opèrent dans les manières d'interagir avec les élèves, mais leurs effets socialisateurs restent réduits. Car les propos des deux enseignantes sont ambivalents : elles adhèrent à cette norme de relation de proximité avec les élèves, mais elles conçoivent toujours leur métier comme une activité au service de la promotion d'une élite obéissante à l'autorité. Inscrites dans un cadre de socialisation assez faible, elles s'approprient partiellement une autre manière d'être enseignant, considérant que le travail quotidien en est grandement facilité, mais y puisant aussi une part de plaisir : " II y a eu beaucoup de changement dans ma personne, dans ma façon de voir les choses. Ce n'est plus l'académique-là qui est privilégié, c'est plus la relation humaine » (Naïma).

\section{Discussion conclusive : poids des configurations locales d'acteurs dans la socialisation professionnelle}

Nos analyses montrent que la pression à l'alignement des pratiques professionnelles est forte. Quand les enseignants formés hors Québec y résistent, leur travail est empêché, par les élèves qui protestent et chahutent et par les parents qui interviennent directement. Les équipes de direction renforcent cette pression, surtout dans une période probatoire où les évaluations négatives peuvent avoir de lourdes conséquences pour les enseignants. Se déploie ainsi une socialisation professionnelle par imposition et obligation, qui a un impact direct et puissant sur les pratiques professionnelles. S'y ajoute un second versant, qui procède tout autrement, par adhésion et compréhension, et vise une appropriation de nouvelles manières de travailler. Cette socialisation s'appuie sur des interactions moins déséquilibrées avec des partenaires de travail qui, en soutenant et accompagnant les 
enseignants formés hors Québec, les initient à la compréhension des situations qu'ils vivent. Les partenaires qui y sont engagés peuvent être nombreux et diversifiés. Nous avons ainsi recensé des acteurs attendus du fait de leurs positions institutionnelles, tels que les responsables hiérarchiques qui dirigent les écoles (et qui suscitent initialement de la crainte tendant à se traduire dans des conduites d'évitement), les experts des difficultés d'apprentissage et de comportement (orthopédagogues, techniciens en éducation spécialisée) avec lesquels les enseignants interagissent d'autant plus facilement qu'ils n'occupent pas de position hiérarchique, ou les autres enseignants, qui apparaissent peu présents et actifs à l'exception de figures singulières avec lesquelles des relations plus personnelles sont nouées. S'y ajoutent d'autres acteurs moins attendus, comme les concierges et secrétaires d'école, qui initient à de multiples règles organisationnelles et avec lesquels des relations plus étroites sont parfois nouées, certains membres des entourages familiaux, particulièrement les enfants qui peuvent faire profiter de leur propre expérience d'élèves au Québec, ou certains élèves, avec qui s'instaure une intercompréhension et qui peuvent délivrer des conseils pour la gestion de tel ou tel problème dans la classe.

Ces configurations d'acteurs ayant un rôle central dans le second type de socialisation sont fortement différenciées selon les contextes de travail. Les effets de ces contextes sur la socialisation sont bien révélés par la comparaison de l'expérience de Rebiha et de celles de ses trois collègues : de multiples partenaires l'ont conseillée, ont porté attention à ses premières expériences, l'ont aidée à décoder les conventions locales. Non seulement elle a adapté ses pratiques sous les contraintes situationnelles auxquelles elle a été rapidement confrontée, mais elle a aussi opérée une véritable " conversion " (Berger et Luckmann, 1986) sur le plan de sa relation avec les élèves et de sa conception de l'activité enseignante. De plus, elle a bénéficié d'un accompagnement privilégié par un mentor informel, une enseignante immigrante déjà " passée par-là ", et donc sachant ce qu'il fallait expliciter de la culture professionnelle locale. À la force de ces soutiens s'ajoute un élément supplémentaire facilitant son intégration : le fait que sa valorisation des performances scolaires, issue de l'expérience d'un système éducatif méritocratique, était en phase avec les objectifs poursuivis par les parents dans le contexte d'une école privée. Elle s'est donc intégrée plus rapidement et facilement, voyant sa maîtrise disciplinaire très valorisée également, ce qui correspond au sens qu'elle accorde au métier.

À contrario, pour Naïma, Anna et Faïza, ce sont des échanges fondés sur des obligations et sanctions qui ont été plus prégnants. Cela a inauguré des processus d'auto-renforcement associant le poids de ce processus de socialisation, l'adoption de positions défensives, le creusement d'une distance avec les partenaires de travail potentiels, le retrait de ces derniers, une faiblesse de la socialisation par compréhension, etc. Loin de s'approprier une nouvelle manière d'enseigner, elles ont surtout adapté leurs pratiques afin de pouvoir malgré tout travailler. Cette dynamique rappelle les stratégies de survie identifiées par Woods (1990) chez les enseignants : la domination (intimider, parler fort, humilier, etc.), la négociation (entrer dans un marchandage avec les élèves qui cherchent à ne pas trop travailler), la fraternisation (se mettre du côté des élèves, se fondre à leur culture pour leur plaire) et le retrait (fuir toute situation qui mettrait en position d'assumer les défis que pose l'enseignement). Le faible engagement de leurs partenaires de travail les prive d'explicitations suffisantes des normes du bien enseigner, ce qui ralentit leur socialisation et la rend chaotique. La fragilité de ces contextes n'est sans doute pas indépendante des conditions d'emploi dans lesquelles elles enseignent, qui les assignent à une inscription précaire dans leurs écoles : fragmentation de la tâche chez Anna, prise en charge d'une classe difficile ayant déjà eu raison de plusieurs enseignants chez Naïma, suppléance à la journée puis petit contrat chez Faïza.

Ces résultats rejoignent, à partir d'un cas spécifique dans un contexte différent, le constat dressé par Dubet d'une faiblesse de la "socialisation institutionnelle "(2002). Dès lors, le modèle de socialisation professionnelle en vigueur s'actualise au cœur des situations de travail, et il est fondé sur une posture réflexive (Périer, 2009). Les épreuves auxquelles les enseignants formés hors Québec, qui s'apparentent sous cet angle à des " nouveaux enseignants " (Rayou et Ria, 2009), font appel à leur réflexivité. Elles signalent aussi la place croissante de la négociation dans le fonctionnement de l'ordre scolaire dans la classe (Périer, 2012) et plus largement dans l'institution scolaire. C'est pourquoi les négociations avec les partenaires de travail sont si importantes pour produire la signification des situations de travail. Mais cet accompagnement est fragile et fluctuant, comme si prévalait une conception implicite des premières années comme ayant pour fonction " d'endurcir le néophyte " (Guibert et Périer, 2012). 
Ces observations invitent à tirer quelque leçon pour les établissements scolaires et pour les commissions scolaires : il faudrait trouver des moyens d'encourager les échanges entre professionnels sur les lieux de travail, de sensibiliser les autres acteurs aux enjeux de socialisation des enseignants formés hors Québec qui débutent dans des contextes nouveaux pour eux, d'inciter les pairs expérimentés à partager leurs expériences et façons de faire quitte à donner plus de porosité à l'espace si protégé de la classe. L'enjeu est de développer une socialisation par compréhension et adhésion, car elle produit des effets plus durables et profonds sur le travail enseignant. Notre hypothèse est que des efforts sur ce plan favoriseraient des adhésions plus rapides et variées aux compréhensions partagées car, comme on l'a vu, lorsque les enseignants arrivent à donner du sens aux pratiques réclamées, ils s'engagent plus aisément dans des processus de transformation de leur rapport au métier.

\section{Références bibliographiques}

Barrère, A. (2002). Les enseignants au travail. Routines incertaines. Paris : L'Harmattan.

Baszanger, I. (1986). Les maladies chroniques et leur ordre négocié. Revue française de sociologie, 27(1), 3-27. http://fapsesrvnt2.unige.ch/Fapse/acra.nsf/AppuisEnseignement/6BA134634FCA7E59C1257C29003CAAE4/\$FILE/Baszanger.pdf

Baszanger, I. (1981). Socialisation professionnelle et contrôle social. Le cas des étudiants en médecine futurs généralistes. Revue française de sociologie, 22(2), 223-245. http://www.persee.fr/doc/rfsoc_00352969_1981_num_22_2_3411

Becker, H. S. (2013). What about Mozart? What about murder? Reasoning from cases. Chicago : The University of Chicago Press.

Becker, H.S. (1963). Outsiders. Study in the sociology of deviance. Glencoe : The Free Press.

Becker, H.S. et McCall, M.M. (dir.). (1990). Symbolic interaction and cultural studies. Chicago, II : University of Chicago Press.

Becker, H.S., Geer, B., Hughes, E.C. et Strauss, A.L. (1961). Boys in white. Student culture in medical school. Chicago, II : University of Chicago Press.

Berger, P. et Luckmann, T. (1986). La construction sociale de la réalité (traduit par P. Taminiaux). Paris : MéridiensKlincksieck.

Berry, J.W. et Sabatier, C. (2011). Variations in the assessment of acculturation attitudes: Their relationships with psychological wellbeing. International Journal of Intercultural Relations, 35(5), 658-669.

Bucher, R. et Strauss, A. (1961). Professions in process. American Journal of Sociology, 66(4), 325-334.

Camilleri, C. (1989). Le choc des cultures. Paris : L'Harmattan.

Charles, F. et Clément, J.-P. (1997). Comment devient-on enseignant ?. Strasbourg : Presses Universitaires de Strasbourg.

Charles, F. et Legendre, F. (2006). Les enseignants issus des immigrations : modalités d'accès au groupe professionnel, représentations du métier et de l'école. Étude comparative. Paris : Sudel.

Cobb, P. et Bauersfeld, H. (1995). Introduction: The coordination of psychological and sociological perspectives in mathematics education. Dans P. Cobb et $\mathrm{H}$. Bauersfeld (dir.), Emergence of mathematical meaning: Interaction in classroom cultures (p. 1-16). Hillsdale, NJ: Lawrence Erlbaum.

Demazière, D. (2011). L'entretien biographique et la saisie des interactions avec autrui. Recherches qualitatives, 30(1), 61-83. http://www.recherche-qualitatve.qc.ca/numero30\%281\%29/RQ_30\%281\%29_Demaziere.pdf

Demazière, D. et Gadéa, C. (2009). La sociologie des groupes professionnels. Paris : La Découverte.

Deters, P. (2006). Immigrant teachers in Canada: Learning the language and culture of a new professional community. Toronto : Ontario Institute for Studies in Education.

Dubet, F. (2002). Le déclin de l'institution. Paris : Seuil. 
Duchesne, C. (2010). À propos de l'accompagnement avant et pendant les stages d'étudiants immigrants inscrits à un programme de formation à l'enseignement. Revue des sciences de l'éducation, 36(1), 95-115. https://www.erudit.org/fr/revues/rse/2010-v36-n1-rse3870/043988ar.pdf

Duchesne, C. (2008). Difficultés d'insertion professionnelle en enseignement : le rôle des conceptions de l'éducation d'étudiants étrangers inscrits dans un programme de formation des maîtres en Ontario. Formation et pratiques d'enseignement en question, 8, 119-139. http://www.revuedeshep.ch/site-fpeq-n/Site_FPEQ/8_files/07_duchesne.pdf

Freidson, E. (1970). Professional Dominance. Chicago, IL : Aldine Publishing Co.

Guignon, S. (2012). La revendication d'un territoire de pratique par des coachs en gestion. Une approche interactionniste pour rendre compte de l'émergence d'un groupe professionnel. Sarrebruck : Les Éditions universitaires européennes.

Guibert, P., Lazuech, G. et Rimbert, F. (2008). Enseignants débutants. "Faire ses classes ». Rennes : Presses universitaires de Rennes.

Guibert, P. et Périer P. (dir.). (2012). La socialisation professionnelle des enseignants du secondaire. Parcours, expériences, épreuves. Rennes: Presses universitaires de Rennes.

Hughes, E.C. (1951). Mistakes at work. Canadian journal of economics and political science, 17(3), 320-327.

Hughes, E.C. (1955). The Making of a Physician. General Statement of Ideas and Problems. Human Organization. 14(4), 21-25.

Jabouin, S. et Duchesne, C. (2012). Le difficile parcours d'insertion des enseignants issus de l'immigration dans la profession enseignante en Ontario. Revue d'éducation de l'Université d'Ottawa, 2(1), 4-5. http://education.uottawa.ca/sites/education.uottawa.ca/files/publication_0.pdf

Kanouté, F. (1999). Les statuts sociométriques et les profils d'acculturation d'élèves d'origine immigrante à Montréal (thèse de doctorat inédite). Université de Montréal, Montréal, Canada.

Laghzaoui, G.A. (2011). Paroles d'immigrants ! Représentations sociales et construction identitaire chez les enseignants immigrants francophones en Colombie-Britannique (thèse de doctorat inédite). Simon Fraser University, Vancouver, Canada.

Lefevbre, M.-L. (2011). L'expérience d'enseignants de groupes minoritaires en milieu scolaire montréalais. Dans J. Tondreau et M. Robert (dir.), L'École québécoise: débats, enjeux et pratiques sociales ( ${ }^{\mathrm{e}}$ éd.) (p. 321-329). Montréal : CEC.

Morrissette, J., Charara, Y., Boily, A. et Diédhiou, B. (2016). Les stratégies des accompagnateurs de l'intégration socioprofessionnelle des enseignants formés à l'étranger: le jeito des despachantes. Revue canadienne de l'éducation, 39(1), 1-29.

Niyubahwe, A., Makamurera, J. et Jutras, F. (2014). L'expérience de transition professionnelle des enseignants de migration récente au Québec. Revue canadienne de l'éducation, 37(4), 1-32. http://journals.sfu.ca/cje/index.php/cje-rce/article/viewFile/1724/1719

Niyubahwe, A., Mukamurera, J., et Jutras, F. (2013). S'adapter au rôle professionnel lorsqu'on est enseignant de migration récente au Québec : expérience, défis et facteurs de réussite. Nouveaux cahiers de la recherche en éducation, 16(2), 56-87. https://www.erudit.org/fr/revues/ncre/2013-v16-n2-ncre01756/1029142ar/

Périer, P. (2009). De l'effacement institutionnel à l'engagement des acteurs. Les professeurs du secondaire entre autonomie et épreuves subjectives. Éducation et Sociétés, 23(1), 27-40.

Périer, P. (2012). L’ordre scolaire dans la classe : une négociation continue. Négociations, 18, 81-92.

Perrenoud, P. (1996). Enseigner : agir dans l'urgence, décider dans l'incertitude. Savoirs et compétences dans un métier complexe. Paris : ESF. 
Phillion, J. (2003). Obstacles to accessing the teaching profession for immigrant women. Multicultural Education, 11(1), 41-46.

Rayou, P. et Ria L. (2009). Former les nouveaux enseignants. Autour des statuts, de l'organisation et des savoirs professionnels. Education et Sociétés, 23(1), 79-90.

Rayou, P. et Van Zanten, A. (2004). Enquête sur les nouveaux enseignants : changeront-ils l'école ?. Paris : Bayard Jeunesse.

Thomas, W.I. (1923). The unadjusted girl. Boston, MA: Brown et Co.

Van Zanten, A. (1999). Les carrières enseignantes dans les collèges difficiles. Dans J. Bourdon et C. Thélot (dir.), Éducation et formation. L'apport de la recherche aux politiques éducatives (p. 99-121). Paris : CNRS Éditions.

Woods, P. (1990). Teacher skills and strategies. London/New York : Falmer Press.

Zietsma, D. (2010). Immigrants exerçant des professions réglementées. Perspective, Février (75-001-X). Ottawa : Statistique Canada. 